\title{
LA TEORÍA DE LAS \\ INTELIGENCIAS MÚLTIPLES ¿PRÁCTICA DOCENTE EN LA EDUCACIÓN DEL PERÚ?
}

\author{
Edica Soledad Jáuregui Salas
}

\begin{abstract}
RESUMEN
El presente artículo parte de una problemática a raíz de los resultados obtenidos en la Evaluación Censal de Estudiantes 2015 (ECE 2015), realizada por la Unidad de Medición de la Calidad Educativa (UMC) del MINEDU. Revela que los alumnos de segundo grado, presentan un desempeño satisfactorio en las áreas de lectura y matemática debajo del $50 \%$. Conlleva a diversas inquietudes en un afán de comprender, analizar qué acciones no se están considerando en la práctica docente, que hace que los educandos no reflejan el interés y el dominio del conocimiento. La teoría de las inteligencias múltiples de Howard Gardner, contribuye a una mirada más amplia de las potencialidades humanas, que por muchos años se mantuvo un concepto estrecho sobre la inteligencia. Asimismo, su implicancia en los contextos escolares.
\end{abstract}

\section{PALABRAS CLAVE}

Inteligencia, inteligencia múltiple, estrategia de enseñanza-aprendizaje.

\section{ABSTRACT}

The present article starts from a problematic one as a result of the results obtained in the Evaluation Cenal de Estudiantes 2015 (ECE 2015), realized by the Unit of Measurement of the Educational Quality (UMC) of the MINEDU. Reveals that second grade students perform satisfactorily in the areas of reading and mathematics below 50\%. It leads to various concerns in an effort to understand, analyze what actions are not being considered in teaching practice, which makes learners do not reflect the interest and mastery of knowledge. Howard Gardner's theory of multiple intelligences contributes to a broader view of human potentialities, which for many years remained a narrow concept of intelligence. Also, its implication in school contexts.

\section{KEYWORDS}

Intelligence, multiple intelligence, teaching - learning strategy.
L

a realidad de la educción en nuestro país, genera honda preocupación en los profesionales docentes, psicólogos educativos, trabajadores sociales y padres de familia que estamos inmersos dentro y fuera de las aulas. Constituye un desafío para el Perú cumplir con los estándares que exige una educación de calidad. Calidad en la enseñanza - aprendizaje, calidad en infraestructura y disponibilidad de la tecnología en los ambientes educativos, sigue siendo una tarea insuficiente, para ver resultados que la sociedad espera de los futuros ciudadanos.

En el año anterior, los datos de la Evaluación Censal de Estudiantes 2015 (ECE 2015) realizada por la Unidad de Medición de la Calidad Educativa (UMC) del MINEDU, reflejan la percepción preocupante por la educación, reveló los siguientes resultados generales en los estudiantes del segundo grado de primaria, en el área de lectura que el 49,8\% mostró un desempeño satisfactorio, $43,8 \%$ en proceso y 6,5\% en inicio; en el área de matemática, que el $26,6 \%$ presentó un desempeño satisfactorio, $42,3 \%$ en proceso y $31,0 \%$ en inicio. Observándose, que los alumnos de segundo grado no alcanzan a obtener un desempeño satisfactorio arriba del 50\% en las áreas de lectura y matemática. Y en relación a ambas áreas evaluadas, el nivel de desempeño satisfactorio es mayor en el área de lectura y menor es el área de matemática. 
Dichos resultados, conlleva a preguntar ¿Qué factores personales o ambientales están influyendo en el desempeño de los educandos? ¿Será la práctica docente poco motivadora para los educandos? ¿Las técnicas utilizadas responden al interés del educando? o ¿El docente tiene un conocimiento actualizado sobre las diferentes formas de enseñar y aprender? ¿Usa el docente las TIC como herramienta para la enseñanza - aprendizaje? Las respuestas a estas interrogantes serán la finalidad del desarrollo del artículo, que propone brindar información, alcances e invitar a la reflexión de la labor docente. La teoría de las inteligencias múltiples de Gardner y su implicancia en la educación, ampliara la visión del potencial humano y como gestionarlo de forma eficaz, promoviendo al aprendizaje significativo, que fortalece al educando y lo prepara para resolver problemas en la vida.

\section{Análisis}

El 28 de julio de 2016, el presidente Pedro Pablo Kuczynski brindó un mensaje a la nación, y con respecto a la educación, planteó que el arte, en especial la música, el deporte y la educación cívica serán materias obligatorias para el año 2017; así como las ciencias, humanidades y el idioma extranjero. Resaltó brindar una educación de calidad, que los educandos entiendan lo que lean, que todos puedan alcanzar estándares internacionales en matemáticas y dominio de un idioma internacional; y que conozcan nuestra cultura milenaria y sus dos grandes idiomas: el quechua y aimara. Invocó a los maestros a ser parte de este sueño.

Atribuir la importancia a la música, al deporte, al idioma y la educación cívica como materias necesarias, es destacar las habilidades y potencias múltiples de las personas. Y mencionar a los docentes en el logro de tal objetivo, es reconocer que son aquellos profesionales que corresponde el gobierno ir de la mano para conseguir el desarrollo del país.

En la medida que las políticas educativas y la práctica docente centren su atención en los intereses y necesidades del educando, con relación al contexto al que pertenecen, se estará consiguiendo los resultados esperados.

Los niños y jóvenes de este siglo XXI pertenecen a la era de la globalización, la tecnología innovadora y de los descubrimientos científicos, tales como nos aporta la neurociencia y la ingeniería genética, así como de las nuevas tendencias sociales, culturales y personales propias de una sociedad cambiante e inestable. Corresponde a los educadores y al estado, entonces responder a la altura de este nuevo escenario. Al respecto, Gardner (2002) señala que los cambios en el mundo son tan rápidos que las escuelas están llamadas a responder a las nuevas demandas de la sociedad con rapidez y de una manera radical, para seguir siendo competitivas, ofreciéndoles una buena educación a la gran mayoría de los futuros ciudadanos.

La teoría de las inteligencias múltiple de Howard Gardner, constituye uno de los grandes aportes para la educación actual, sobre el tema de la inteligencia, las inteligencias múltiples, proporcionando una herramienta de apoyo en beneficio de los educandos, particularmente, dentro del proceso de enseñanza aprendizaje.

Plantea una visión amplia sobre la competencia intelectual humana; ya que, hasta antes de la década de los 80, se tenía un concepto demasiado estrecho, que la inteligencia de un individuo se basaba en la medición de un CI (Cociente intelectual), basado predominante en las capacidades lingüística y matemática.

Gardner $(1994,1999)$, ha propuesto su teoría de las Inteligencias Múltiples, en la que sus fundamentos teóricos están basados en la valoración de las capacidades del individuo y en la importancia de expresar que la inteligencia es la capacidad para resolver problemas cotidianos, generar nuevos problemas, crear productos y ofrecer servicios dentro del propio ámbito cultural (Suarez, Maíz y Meza 2010: 10).

Hasta la actualidad, ha identificado hasta ocho tipos distintos de inteligencias, ampliándose de esta manera los alcances del potencial humano, que en resumen se menciona: 


\begin{tabular}{|l|l|}
\hline \multicolumn{1}{|c|}{ Inteligencia } & \multicolumn{1}{|c|}{ Descripción } \\
\hline 1. Lingüistica & $\begin{array}{l}\text { Un buen desarrollo en destrezas verbales y en la } \\
\text { sensibilidad a los sonidos, significados y ritmos de } \\
\text { las palabras. }\end{array}$ \\
\hline 2. Lógico-Matemática & $\begin{array}{l}\text { Aptitud para pensar conceptual y abstractamente y } \\
\text { capacidad para discernir patrones lógicos o } \\
\text { numéricos. }\end{array}$ \\
\hline 3. Visual -espacial & $\begin{array}{l}\text { Capacidad de pensar en imágenes y dibujos, } \\
\text { visualizar fiel y abstractamente. }\end{array}$ \\
\hline 4. Musical & $\begin{array}{l}\text { Aptitud para producir y apreciar el ritmo, tono y } \\
\text { timbre. }\end{array}$ \\
\hline 5. Manual -Kinésica & $\begin{array}{l}\text { Aptitud de manipular objetos hábilmente y } \\
\text { controlar los movimientos del propio cuerpo. }\end{array}$ \\
\hline 6. Interpersonal & $\begin{array}{l}\text { Capacidad de detectar y responder } \\
\text { apropiadamente a los modos, motivaciones y } \\
\text { deseos de otros. }\end{array}$ \\
\hline 7. Intrapersonal & $\begin{array}{l}\text { Capacidad de autoconciencia y de estar en } \\
\text { sintonía con los sentimientos intimos, valores, } \\
\text { creencias y procesos de pensamiento. }\end{array}$ \\
\hline 8. Naturalista & $\begin{array}{l}\text { Aptitud de reconocer y categorizar plantas, } \\
\text { animales y otros objetos de la naturaleza. }\end{array}$ \\
\hline
\end{tabular}

Inclusive está considerando la posibilidad de una novena inteligencia, la inteligencia existencial, que podría definirse como la sensibilidad y capacidad para analizar en sentido más amplio y a profundidad cuestionamientos acerca de la existencia humana, como el significado de la vida, por qué morimos y cómo llegamos aquí (Bilbao y Velasco 2015).

Algunas aseveraciones de Gardner (1994, 1999), enfatizan el hecho de que todas las inteligencias son igualmente importantes (...). Todas las personas tienen la capacidad de desarrollar las inteligencias a un nivel relativamente alto. Lo importante es tener la motivación y la instrucción adecuada (Suarez et al. 2010, párr. 20).

Inteligencias múltiples en las aulas, una propuesta de estilos para enseñar y aprender

La teoría de las inteligencias múltiples, ofrece a los docentes a tomar conciencia sobre la naturaleza y la calidad de sus diferentes tipos de inteligencia y como desarrollarlos, además, de analizar de qué manera influye en el desempeño de su rol como educadores. Asimismo, ayuda a los docentes a expandir sus estrategias pedagógicas que le garanticen un aprendizaje significativo en sus educandos.

Armstrong (2007), señala que el aporte de la teoría de la IM a la educación es sugerir que los docentes amplíen el repertorio de técnicas, herramientas y estrategias más allá de las que aplican en las aulas, predominantemente lingüísticas y lógicas. Además, Matos (2012) señala:
(...), en los niveles de inicial y primaria, esta teoría aporta de manera significativa herramientas para favorecer el estímulo de las inteligencias menos desarrolladas y fortalecer aquellas que han sido mejor logradas. En el nivel secundario, se continúa orientando la metodología en función a la estimulación de las múltiples inteligencias pero se pone énfasis en la mirada vocacional del individuo como parte de la construcción de su proyecto de vida que le permita forjarse un futuro más seguro para la supervivencia y mejora de su calidad de vida (p.17).

Reconocer la existencia de diferentes tipos de inteligencia, plantea considerar ocho estilos de enseñar y de aprender. "Las asignaturas pueden presentarse de formas muy diversas que permitan al estudiante asimilarlas partiendo de sus capacidades y aprovechando sus puntos fuertes" (Suarez et al. 2010, párr. 26).

Armstrong (2007, afirma: El docente en un aula de IM se diferencia de manera marcada del docente en un aula tradicional. En el aula tradicional, el docente da una clase parado en el frente del aula, escribe en el pizarrón, hace preguntas a los alumnos sobre las lecturas o tareas asignadas y espera que los alumnos terminen su trabajo escrito. En el aula de IM, el docente cambia todo el tiempo su método de presentación, del campo lingüístico al especial, al musical y así como todas las demás inteligencias, combinándolas, a menudo, de maneras creativas ( $\mathrm{p}$. 76).

Bilbao y Velasco (2015) refieren que "la teoría de las inteligencias múltiples construye una buena enseñanza - aprendizaje dentro del currículo existente, al expandir el número de formas en que los educandos pueden representar sus aprendizajes y conocimientos del tema que van estudiando" (p. 82). Asimismo, "esta teoría también implica la esperanza de motivar con mayor efectividad a más alumnos, al tener en cuenta sus métodos predilectos de adquisición de conocimiento en los planes curriculares, en el proceso de enseñanza y en la evaluación" (Brunner y Rottensteiner 2006: 25).

Guillen (2013), indica que la teoría de las inteligencias múltiples impulsa un aprendizaje por proyectos por parte de los alumnos que facilita a una mejor comprensión de los contenidos. Constituyen una alternativa a los exámenes tradicionales porque 
el progreso de su aprendizaje se evalúa analizando el avance de su desempeño. Este proceso de aprendizaje se puede documentar en registros o portafolio evaluativo (trabajos escritos, dibujos, pinturas, videos, presentaciones en computadora, etc.) que muestren la participación del educando en los diferentes proyectos o actividades. Todo en coherencia con el aprendizaje significativo en el que el currículo y la evaluación están integrados.

La tecnología y el enfoque de las inteligencias múltiples

Actualmente, los niños están creciendo en un mundo digital, la tecnología es parte de sus vidas. Y para los docentes, constituye un nuevo rol, son los llamados a preparar a los educandos para enfrentarse al mundo de hoy, por lo que han de tener un conocimiento exhaustivo de este, y por lo que su desarrollo profesional debe evolucionar a medida que avanza la sociedad.

Bilbao y Velasco (2015), señalan: Internet puede ofrecer una riqueza de recursos que permite a los educandos experimentar con la información que se presenta en numerosos formatos: presentación de diapositivas, animación interactiva, simulación, sonidos, gráficos, video y texto (p. 83).

Armstrong (2007), señala que la funcionalidad de las computadoras está en los programas de software. Y estos programas pueden diseñarse para interactuar con cualquiera de las inteligencias múltiples o con todas ellas. Por ejemplo, existen los programas de procesamiento de texto, que exige utilizar la inteligencia lingüística, o los programas para dibujar y pintar, que pone en práctica la inteligencia espacial. Hay una variedad de programas software disponible para estimular las inteligencias múltiples, y ponerlos a disposición de los educandos en el laboratorio de la escuela, es favorecer a que los educadores amplíen sus estrategias de enseñanza-aprendizaje.

En el cuadro siguiente, Bilbao y Velasco (2015) mencionan algunos programas disponibles para los ambientes educativos, que activan diferentes áreas de la inteligencia:

\begin{tabular}{|c|c|}
\hline Inteligencia & Herramientas \\
\hline $\begin{array}{l}\text { Capacidad para usar el lenguaje, la lengua } \\
\text { materna y tal vez otras lenguas, con objeto } \\
\text { de expresar lo que hay en nuestra mente y } \\
\text { entender a otras personas. }\end{array}$ & $\begin{array}{l}\text { - Programas para procesar textos. } \\
\text { - Herramientas para presentaciones } \\
\text { multimedia. } \\
\text { - Programas para lenguas extranjeras. } \\
\text { - CD, DVD, Blu-ray de libros de } \\
\text { cuentos. } \\
\text { - Programas de juegos con palabras. } \\
\text { - Blogs. }\end{array}$ \\
\hline $\begin{array}{l}\text { Lógico-matemática } \\
\text { Personas con un alto desarrollo en esta } \\
\text { inteligencia entienden los principios } \\
\text { subyacentes de algún tipo casual, a la } \\
\text { manera en que lo hace un cientifico o un } \\
\text { especialista en logística; o que puede } \\
\text { manipular números, cantidades y } \\
\text { operaciones, como lo hacen los } \\
\text { matemáticos. }\end{array}$ & $\begin{array}{l}\text { - Tutoriales en destrezas matemáticas. } \\
\text { - Herramientas para elaborar mapas. } \\
\text { - Juegos de lógica. } \\
\text { - Programas para resolver problemas. } \\
\text { - CD, DVD, Blu-ray de libros. } \\
\text { - Hojas de cálculo. } \\
\text { - Tutores en programación de } \\
\text { computadoras. }\end{array}$ \\
\hline $\begin{array}{l}\text { Visual-espacial } \\
\text { Habilidad para representar el mundo } \\
\text { espacial en nuestra mente. Puede utilizarse } \\
\text { en las artes o en las ciencias. }\end{array}$ & $\begin{array}{l}\text { - Programas de animación. } \\
\text { - Lenguajes de modelación en tercera } \\
\text { dimensión. } \\
\text { - Programa de galería multimedia. } \\
\text { - (Clip Art) } \\
\text { - Cámaras digitales y microscopios. } \\
\text { - Programas para dibujar y pintar. } \\
\text { - Equipos de rompecabezas electrónicos. } \\
\text { - Juegos para resolver problemas } \\
\text { espaciales. }\end{array}$ \\
\hline $\begin{array}{l}\text { Musical } \\
\text { Capacidad para pensar en forma musical, } \\
\text { ser capaz de escuchar patrones, } \\
\text { reconocerlos y tal vez manipularlos. }\end{array}$ & $\begin{array}{l}\text { - Tutores en documentos musicales. } \\
\text { - Programas para cantar (sintetizador de } \\
\text { voz). } \\
\text { - Potenciadores de melodía y de } \\
\text { reconocimiento de tono. } \\
\text { - Programas para creación de música. } \\
\text { - CD, DVD, Blu-ray de música. }\end{array}$ \\
\hline $\begin{array}{l}\text { Manual -kinésica } \\
\text { Capacidad para utilizar todo el cuerpo o } \\
\text { partes de él, para resolver un problema, } \\
\text { efectuar una tarea o presentar alguna clase } \\
\text { de producción. }\end{array}$ & $\begin{array}{l}\text { - Juegos de coordinación vasomotora. } \\
\text { - Tabletas electrónicas. } \\
\text { - Consolas de videojuegos (Wii, } \\
\text { Kinect) }\end{array}$ \\
\hline $\begin{array}{l}\text { Interpersonal } \\
\text { Comprensión hacia otras personas. }\end{array}$ & $\begin{array}{l}\text { - Juegos de simulación. } \\
\text { - } \quad \text { Programa de correos electrónicos. } \\
\text { - } \quad \text { Roros de discusión. } \\
\text { Moodle, gociales (Facebook, twitter, } \\
\text { Moogl }\end{array}$ \\
\hline $\begin{array}{l}\text { Intrapersonal } \\
\text { Poseer el entendimiento de uno mismo, } \\
\text { saber quiénes somos, lo que podemos } \\
\text { hacer, lo que queremos hacer, cómo } \\
\text { reaccionamos ante las situaciones, qué } \\
\text { evitar y hacia cuáles dirigirnos. }\end{array}$ & $\begin{array}{l}\text { - } \quad \text { Programas de elección personal. } \\
\text { - } \quad \text { Programas de orientación profesional. } \\
\text { - } \quad \text { Cualquier programa autodidáctico. }\end{array}$ \\
\hline $\begin{array}{l}\text { Capacidad humana para discriminar entre } \\
\text { seres vivos, así como sensibilidad hacia } \\
\text { otras características del mundo natural. }\end{array}$ & $\begin{array}{l}\text { - } \begin{array}{l}\text { Archivos de sonido o imágenes de la } \\
\text { naturaleza. } \\
\text { - Programas de clasificación } \\
\text { flora/fauna. } \\
\text { - Programas para identificar sonidos de } \\
\text { animales. } \\
\text { - Programa de ciencia de la tierra. } \\
\text { - Páginas de Internet relacionados con } \\
\text { el tema. }\end{array} \\
\end{array}$ \\
\hline
\end{tabular}

\section{CONCLUSIONES}

1. El Ministerio de Educación, ente que planifica y dirige las políticas educativas y los docentes, tiene la tarea de trabajar de forma coordinada, integrada y contextualizada, respondiendo a las necesidades 
y demandas del educando de hoy. Docentes actualizados en los avances de la educación, psicología y en neurociencia, así como mostrar dominio en TIC, deben conformar el perfil del educador, que los niños y jóvenes de hoy demandan.

2. La teoría de las inteligencias múltiples, es un modelo que corresponde al enfoque del aprendizaje constructivista. Aporta un sinfín de herramientas al docente de hoy, cuya finalidad es promover el aprendizaje significativo en los educandos.

3. Con la teoría de la inteligencia múltiple comienza una nueva concepción de la inteligencia. Howard Gardner propone ocho tipos de inteligencia: lógico-matemática, lingüístico-verbal, corporalkinestésica, espacial, musical, interpersonal, intrapersonal y naturalista y todos estamos en la posibilidad de desarrollarlas aunque de un modo y a un nivel particular resultado de la dotación biológica, pero en particular de la interacción con el entorno y la cultura, que las promueven o inhiben.

4. Todas las inteligencias son importantes, una educación basada en la valoración de las capacidades individuales, favorecerá en el proceso enseñanza - aprendizaje del educando cada vez más heterogéneo.

\section{REFERENCIAS}

Bilbao Rodríguez, María del Carmen y Velasco García, Patricia (2015). Aprendizajes con inteligencias múltiples. Cómo identificar las inteligencias múltiples, cómo desarrollarlas y cómo evaluarlas. México: Trillas.

Brunner, Ilse y Rottensteiner, Erika (2006). El desarrollo de las inteligencias en la infancia. Ejemplos prácticos para una enseñanza exitosa. D. F., México: Fondo de Cultura Económica.

Evaluación Censal de Estudiantes 2015. 2o grado de educación primaria. http://umc.minedu.gob.pe /wp-content/uploads/2016/03/Resultados-ECE2015.pdf

Gardner, Howard (2002). La educación de la mente y el conocimiento de las disciplinas. Lo que todos los estudiantes deberían comprender. Barcelona, España: Paidos.

Gadner, Howard (1995). Estructuras de la mente. La teoría de las inteligencias múltiples. México: Fondo de Cultura Económica.
5. Conocer los docentes sus propias potencialidades intelectuales, les permite reflexionar de qué modo influye en su desempeño y asimismo, ayudar a los educandos a reconocer las fortalezas que tienen y de los aspectos que necesita desarrollar para favorecer en su aprendizaje.

6. La teoría de las inteligencias múltiples nos enseña a aprovechar con mayor efectividad las habilidades humanas, propone una variedad de herramientas y estrategias para enseñar y aprender, que garantiza el aprendizaje significativo deleducando.

7. Tomar en cuenta los métodos predilectos de adquisición de conocimiento en los planes curriculares, en el proceso de enseñanza y en la evaluación de los educandos, permite tomar en cuenta factores afectivos como la motivación y autoestima, que también es importante para construir un aprendizaje significativo.

8. La tecnología constituye una herramienta que permite desarrollar los diferentes tipos de inteligencia. Es importante que los laboratorios de computación cuenten con una variedad de programas de software que activan diferentes áreas de la inteligencia. Los educandos de hoy, son nativos digitales, exigen entonces al docente estar a la medida que avanza la sociedad.

Guillen, Jesús C. (2013). Inteligencias múltiples en el aula. https://escuelaconcerebro.wordpress. com/2013/05/05/inteligencias-multiples-en-elaula/Mayo2013.

Matos Caparó, Félix M. (2012). Inteligencias múltiples en estudiantes de tercer grado de secundaria de una institución educativa de Ventanilla - Callao. http://repositorio.usil.edu.pe/bitstream/123456789/ 1183/1/2012_Matos_Inteligencias $\% 20 \mathrm{~m} \%$ C3\%BAltiple s\%20en $\% 20$ estudiantes $\% 20$ de $\% 20$ tercer $\% 20$ grado $\%$ 20de\%20secundaria\%20de\%20una\%20instituci\%C3 \%B3n\%20educativa\%20de\%20Ventanilla-Callao.pdf

Suárez, Jacqueline; Maiz, Francelysy; Meza, Marina (2010). Inteligencias múltiples: Una innovación pedagógica para potenciar el proceso enseñanza aprendizaje. http://www.scielo.org.ve/scielo. php? script=sci_arttext\&pid=S131600872010000100005 Äbril 2010. 